\title{
TWISTED SEQUENCES OF EXTENSIONS
}

\author{
KEVIN J. CARLIN
}

\begin{abstract}
Gabber and Joseph [GJ, §5] introduced a ladder diagram between two natural sequences of extensions. Their diagram is used to produce a 'twisted' sequence that is applied to old and new results on extension groups in category $\mathcal{O}$.
\end{abstract}

\section{The GabBer-Joseph Isomorphism}

Let $\mathcal{A}$ be an abelian category with enough projectives. Let $\mathrm{E}^{p}=\operatorname{Ext}_{\mathcal{A}}^{p}$ (with the convention that $\mathrm{E}^{p}=0$ if $p<0$ ). Let $\mathrm{H}=\mathrm{E}^{0}=\operatorname{hom}_{\mathcal{A}}$. If $\mathrm{E}$ is used to represent some $\mathrm{E}^{p}$, then use the relative notations, $\mathrm{E}^{+}$and $\mathrm{E}^{-}$, to represent $\mathrm{E}^{p+1}$ and $\mathrm{E}^{p-1}$ respectively.

Suppose that $R$ and $T$ are exact, mutually adjoint endofunctors defined on $\mathcal{A}$. Let $\theta=R T$. The unit of the adjunction $(T, R)$ is $\eta: \mathrm{Id} \rightarrow \theta$ and the co-unit of the adjunction $(R, T)$ is $\epsilon: \theta \rightarrow \mathrm{Id}$. Use these to define the functors,

$$
\begin{aligned}
C & =\text { Coker } \eta & D & =\operatorname{Coim} \eta \\
K & =\operatorname{Ker} \epsilon & I & =\operatorname{Im} \epsilon .
\end{aligned}
$$

There are also natural transformations, $\iota: I \rightarrow \operatorname{Id}$ and $\pi: \operatorname{Id} \rightarrow D$.

There is a natural adjoint pairing $(C, K)$ so that $C$ is right exact and $K$ is left exact. If $M$ and $N$ are objects in $\mathcal{A}$, there are canonical exact sequences, $K N \hookrightarrow \theta N \rightarrow I N$ and $D M \hookrightarrow \theta M \rightarrow C M$. Each gives rise to a long exact sequence of extensions.

Theorem 1.1 [GJ, 5.1.8] Suppose that $M$ is C-acyclic. There is a natural commutative diagram with exact rows,

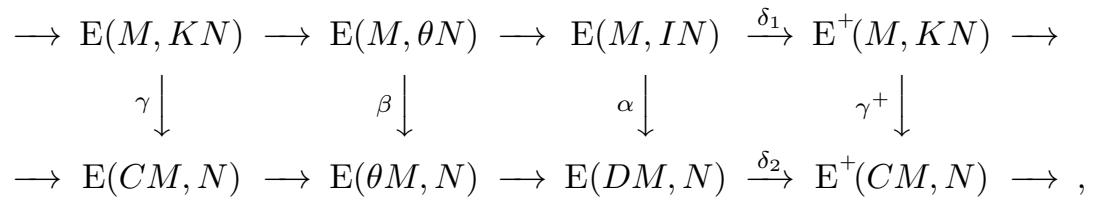

where $\beta$ is an isomorphism. If $D M$ is $C$-acyclic and $I N=N$, then $\alpha$ and $\gamma$ are isomorphisms.

Proof. Let $P \rightarrow M$ be a projective resolution. There is an exact sequence of chain complexes,

$$
0 \longrightarrow D P \longrightarrow \theta P \longrightarrow C P \longrightarrow 0
$$

2010 Mathematics Subject Classification. 17B10, 17B55. 
Since $M$ is $C$-acyclic, this is a resolution of the exact sequence,

$$
0 \longrightarrow D M \longrightarrow \theta M \longrightarrow C M \longrightarrow 0 .
$$

Let $X \rightarrow D M$ and $Z \rightarrow C M$ be projective resolutions. Use the horseshoe lemma $[\mathrm{W}, 2.28]$ to construct a split exact sequence resolving diagram (1.1.1),

$$
0 \longrightarrow X \rightarrow Y \rightarrow Z \longrightarrow 0 \text {. }
$$

By the comparison theorem [W, 2.3.7], there are chain maps $a: X \rightarrow D P$ and $c: Z \rightarrow C P$ lifting $\operatorname{Id}_{D M}$ and $\operatorname{Id}_{C M}$ respectively. Using the splitting maps of diagram (1.1.2), construct a chain map $b: Y \rightarrow \theta P$ lifting $\operatorname{Id}_{\theta M}$ and completing a commutative diagram of chain complexes with exact rows,

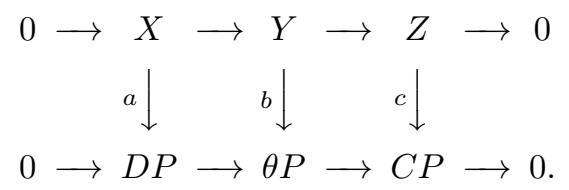

Applying $\mathrm{H}(-, N)$ yields a commutative diagram with exact rows,

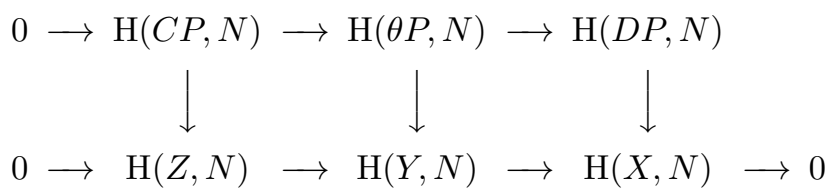

Since $P$ is a projective complex, there is also a natural commutative diagram of complexes with exact rows,

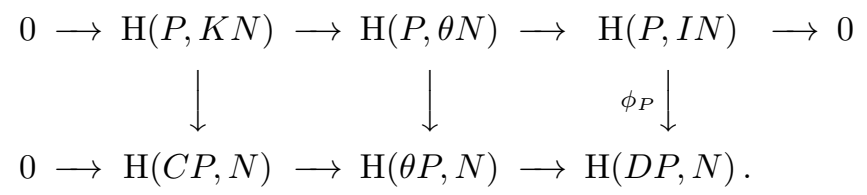

The chain map $\phi_{P}$ is uniquely defined by the diagram because $\mathrm{H}\left(\pi_{P}, N\right) \phi_{P}=$ $\mathrm{H}\left(P, \iota_{N}\right)$. The first two vertical mappings are isomorphisms.

Combining diagram (1.1.3) and diagram (1.1.4), and applying [W, 1.3.4] yields the Gabber-Joseph diagram. Since $\theta P \rightarrow \theta M$ is a projective resolution, $b$ is a homotopy equivalence so $\beta$ is an isomorphism. (So far, this is the same as the proof given in [GJ, 5.1.8].)

Let $f: P \rightarrow X$ be a chain map lifting $\pi_{M}$. Then, by the uniqueness part of the comparison theorem, af is homotopic to $\pi_{P}$. So, $\mathrm{H}(f, N) \mathrm{H}(a, N) \phi_{P}$ is homotopic to $\mathrm{H}\left(\pi_{P}, N\right) \phi_{P}=\mathrm{H}\left(P, \iota_{N}\right)$. Passing to cohomology, $\mathrm{E}\left(\pi_{M}, N\right) \alpha=\mathrm{E}\left(M, \iota_{N}\right)$.

Now suppose that $D M$ is $C$-acyclic so that $D X \rightarrow D M$ is a resolution. The chain map $D(f): D P \rightarrow D X$ lifts $\operatorname{Id}_{D M}$ so $D(f) a$ is homotopic to $\pi_{X}$. Hence $\mathrm{H}(a, N) \mathrm{H}(D(f), N) \phi_{X}$ is homotopic to $\mathrm{H}\left(\pi_{X}, N\right) \phi_{X}=\mathrm{H}\left(X, \iota_{N}\right)$.

By functoriality, $\mathrm{H}(f, N) \mathrm{H}\left(X, \iota_{N}\right)=\mathrm{H}\left(P, \iota_{N}\right) \mathrm{H}(f, I N)$ and, since $\pi$ is a natural transformation, $\mathrm{H}\left(\pi_{P}, N\right) \mathrm{H}(D(f), N)=\mathrm{H}(f, N) \mathrm{H}\left(\pi_{X}, N\right)$. Then,

$$
\begin{aligned}
\mathrm{H}\left(\pi_{P}, N\right) \mathrm{H}(D(f), N) \phi_{X} & =\mathrm{H}(f, N) \mathrm{H}\left(\pi_{X}, N\right) \phi_{X}=\mathrm{H}(f, N) \mathrm{H}\left(X, \iota_{N}\right) \\
& =\mathrm{H}\left(P, \iota_{N}\right) \mathrm{H}(f, I N)=\mathrm{H}\left(\pi_{P}, N\right) \phi_{P} \mathrm{H}(f, I N) .
\end{aligned}
$$


Since $\mathrm{H}\left(\pi_{P}, N\right)$ is a monomorphism, $\mathrm{H}(D(f), N) \phi_{X}=\phi_{P} \mathrm{H}(f, I N)$ which means that $\mathrm{H}(a, N) \phi_{P} \mathrm{H}(f, I N)$ is homotopic to $\mathrm{H}\left(X, \iota_{N}\right)$. Passing to cohomology yields $\alpha \mathrm{E}\left(\pi_{M}, I N\right)=\mathrm{E}\left(D M, \iota_{N}\right)$.

If $I N=N, \mathrm{E}\left(M, \iota_{N}\right)=\mathrm{Id}$ and $\mathrm{E}\left(D M, \iota_{N}\right)=\mathrm{Id}$, so that $\alpha$ is an isomorphism. By the long-five lemma, $\gamma$ is also an isomorphism.

Corollary 1.2 If $M$ and $D M$ are $C$-acyclic, then $\mathrm{E}(M, K N)$ and $\mathrm{E}(C M, I N)$ are isomorphic.

Proof. By standard properties of adjunction maps, $T\left(\epsilon_{N}\right)$ is an epimorphism. So $I\left(\iota_{N}\right)$ is an isomorphism as are $\theta\left(\iota_{N}\right)$ and $K\left(\iota_{N}\right)$. In this way, $I(I N), \theta(I N)$, and $K(I N)$ will be identified with $I N, K N$, and $\theta N$ respectively. Applying theorem 1.1 to $I N$, there is a commutative diagram,

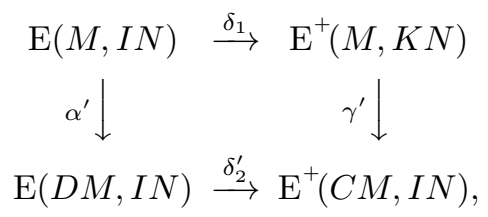

where the vertical mappings are isomorphisms and the primes indicate maps defined with respect to $I N$.

\section{The Twisted Sequence}

Theorem 2.1 Suppose that $M$ and DM are $C$-acyclic. There is a commutative diagram with exact rows,

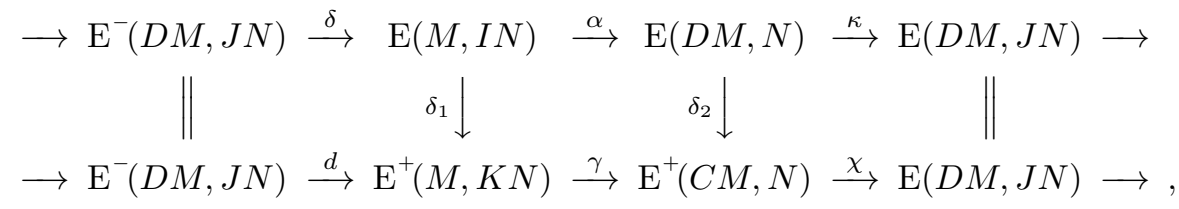

where $J N=$ Coker $\epsilon_{N}$. If $D M=M$, the first row is the long exact sequence associated to the exact sequence, $I N \hookrightarrow N \rightarrow J N$.

Proof. Let $\phi_{P}^{\prime}$ be the map defined by (1.1.4) with $N=I N$. Then $\mathrm{H}\left(\pi_{P}, I N\right) \phi_{P}^{\prime}=\mathrm{Id}$. Using the notation from the previous section,

$$
\begin{aligned}
\mathrm{H}\left(\pi_{P}, N\right) \mathrm{H}\left(D P, \iota_{N}\right) \phi_{P}^{\prime} & =\mathrm{H}\left(P, \iota_{N}\right) \mathrm{H}\left(\pi_{P}, I N\right) \phi_{P}^{\prime} \\
& =\mathrm{H}\left(P, \iota_{N}\right)=\mathrm{H}\left(\pi_{P}, N\right) \phi_{P} .
\end{aligned}
$$

Because $\mathrm{H}\left(\pi_{P}, N\right)$ is a monomorphism, $\mathrm{H}\left(D P, \iota_{N}\right) \phi_{P}^{\prime}=\phi_{P}$. Then

$$
\mathrm{H}(a, N) \phi_{P}=\mathrm{H}(a, N) \mathrm{H}\left(D P, \iota_{N}\right) \phi_{P}^{\prime}=\mathrm{H}\left(X, \iota_{N}\right) \mathrm{H}(a, I N) \phi_{P}^{\prime} .
$$

Taking cohomology, $\alpha=\mathrm{E}\left(D M, \iota_{N}\right) \alpha^{\prime}$. In a similar fashion, $\beta=\mathrm{E}\left(\theta M, \iota_{N}\right) \beta^{\prime}$ and $\gamma=\mathrm{E}\left(C M, \iota_{N}\right) \gamma^{\prime}$. 
Diagram 1:

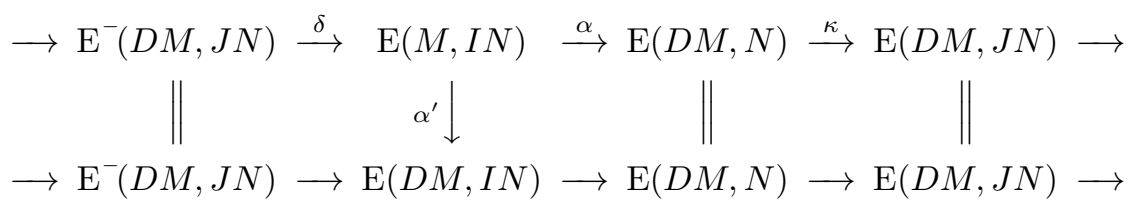

The second row is the long exact sequence associated to $I N \hookrightarrow N \rightarrow J N$. Since $\alpha^{\prime}$ is an isomorphism, define $\delta$ so that the first square commutes. This produces a commutative diagram with exact rows. If $D M=M, \alpha^{\prime}=\mathrm{Id}$ which proves the second conclusion.

Diagram 2:

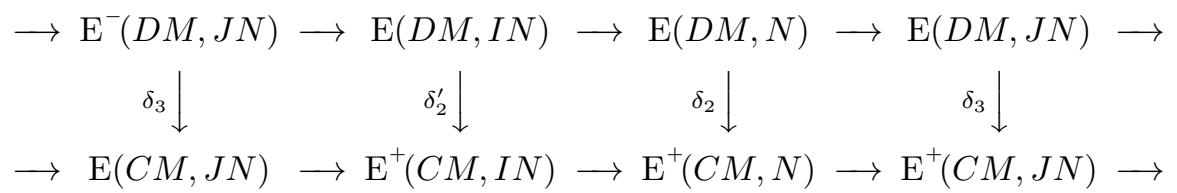

This is a commutative diagram with exact rows where the vertical maps are the natural connecting maps.

\section{Diagram 3:}

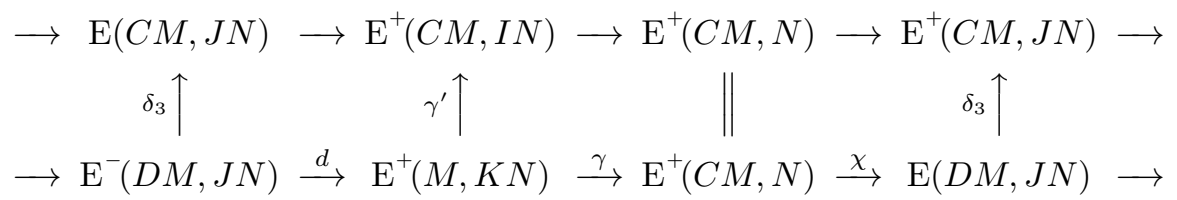

Since $T\left(\epsilon_{N}\right)$ is surjective, $T J N=0$. By the adjoint pairing $(R, T), \mathrm{E}(\theta M, J N)=$ $\mathrm{E}(T M, T J N)=0$ so $\delta_{3}$ is an isomorphism. Define $d$ and $\chi$ to make the diagram commutative. Then the second row is also exact.

Assembling the three diagrams proves the first conclusion since $\delta_{3}^{-1} \delta_{3}=\mathrm{Id}$ and $\left(\gamma^{\prime}\right)^{-1} \delta_{2}^{\prime} \alpha^{\prime}=\delta_{1}$.

The second row of 2.1 will be referred to as a twisted sequence.

\section{Applications in CATEgory $\mathcal{O}$ : older Results}

Let $\mathfrak{g}$ be a finite-dimensional semisimple Lie algebra over $\mathbb{C}$. Category $\mathcal{O}$ is the category of $\mathfrak{g}$-modules introduced in $[\mathrm{BGG}]$. For background information on category $\mathcal{O}$, we will rely on [Hum2] where the original sources and the later developments can be found.

Let $S$ be the set of simple root reflections in the Weyl group $W$. The stabilizer of a weight $\lambda$ under the dot action is $W_{\lambda}^{\circ}$. Let $w_{0}$ denote the longest element and let 1 denote the identity. The Bruhat order on $W$ is denoted by $<$. Let $\xi$ be its 
characteristic function defined by

$$
\xi(x, y)= \begin{cases}1 & \text { if } x \leq y \text { and } \\ 0 & \text { otherwise }\end{cases}
$$

Let $\ell$ denote the length function on $W$. If $x, y \in W, \ell(x, y)=\ell(y)-\ell(x)$.

The $R$-polynomials are defined in [Hum1, $\S 7]$. Let $r_{p}(x, y)$ denote the coefficient of $q^{p}$ in $(-1)^{n-p} R_{x, y}$ where $n=\ell(x, y)$. A recursion for $r_{p}(x, y)$ begins with $r_{p}\left(w_{0}, y\right)=0$ if $p \neq 0$ and $r_{0}\left(w_{0}, y\right)=\xi\left(w_{0}, y\right)$. If $x<w_{0}$, choose an $s \in S$ so that $x s>x$. Then, for all $p$,

$$
r_{p}(x, y)= \begin{cases}r_{p}(x s, y s) & \text { if } y s>y \\ r_{p}(x s, y)+r_{p-1}(x s, y)-r_{p-1}(x s, y s) & \text { if } y s<y\end{cases}
$$

The following properties of the $r_{p}$ can be proved by induction or translated from properties of the $R$-polynomials in [Hum1, §7]. If $r_{p}(x, y) \neq 0$, then $x \leq y$ and $0 \leq p \leq \ell(x, y)$. Also $r_{0}=\xi$ and, if $n=\ell(x, y), r_{p}(x, y)=r_{n-p}(x, y)$.

Specializing $(3.0 .1)$ to $p=1, r_{1}\left(w_{0}, y\right)=0$ and, if $x s>x$,

$$
r_{1}(x, y)= \begin{cases}r_{1}(x s, y s) & \text { if } y s>y, \\ r_{1}(x s, y)+1 & \text { if } y s<y \text { and } x s \nless y s, \\ r_{1}(x s, y) & \text { if } y s<y \text { and } x s<y s .\end{cases}
$$

Choose anti-dominant integral weights $\lambda$ and $\mu$ so that $W_{\lambda}^{\circ}=\{e\}$ and $W_{\mu}^{\circ}=\{e, s\}$ where $s \in S$. If $x \in W$, let $M_{x}$ denote the Verma module with highest weight $x \cdot \lambda$. The block of $\mathcal{O}$ with projective generator $M_{w_{0}}$ is $\mathcal{O}_{\lambda}[\mathrm{Hum} 2,4.9]$. Here, $T$ is the translation functor $T_{\lambda}^{\mu}$ where $R$ is its left and right adjoint $T_{\mu}^{\lambda}$ [Hum2, 7.1-2]. A module $M \in \mathcal{O}_{\lambda}$ is $C$-acyclic if, and only if, $D M=M[\mathrm{C}, 2.9]$ and this condition is true for each $M_{x}[\mathrm{C}, 2.8(\mathrm{i})]$.

For $x, y \in W$, write $\mathrm{E}^{p}(x, y)$ for $\mathrm{E}^{p}\left(M_{x}, M_{y}\right)$ and $e_{p}(x, y)$ for its dimension. Also, for $x$ and $z \leq y$ in $W$, write $\mathrm{E}^{p}(x, y / z)$ for $\mathrm{E}^{p}\left(M_{x}, M_{y} / M_{z}\right)$ and let $e_{p}(x, y / z)$ be the dimension.

Since $M_{w_{0}}$ is projective, $e_{p}\left(w_{0}, y\right)=0$ if $p \neq 0$. By the properties of homomorphisms between Verma modules, $e_{0}=\xi\left[\right.$ Hum2, 5.2], so $e_{0}=r_{0}$. The vanishing properties also match. If $e_{p}(x, y) \neq 0$ then $x \leq y$ and $0 \leq p \leq \ell(x, y)$ [Hum2, 6.11].

The twisted sequence can be used to re-prove some of the results of [GJ, 5.2].

Proposition 3.1 [GJ, 5.2.1] Suppose that $x s>x$ and $y s<y$. For all $p$,

$$
e_{p}(x s, y)=e_{p}(x, y s) \text {. }
$$

Proof. Let $M=M_{x s}$ and $N=M_{y s}$. Then $C M=M_{x}, I N=N$, and $K N=M_{y}[\mathrm{C}$, 3.5]. By 1.2, $\mathrm{E}(x s, y)$ is isomorphic to $\mathrm{E}(x, y s)$.

Suppose that $x s>x$ and $y s<y$. Apply 2.1 with $M=M_{x s}$ and $N=M_{y}$. Then $I N=M_{y s}, C M=M_{x}$, and $K N=M_{y}$. There is a commutative diagram with exact 
rows,

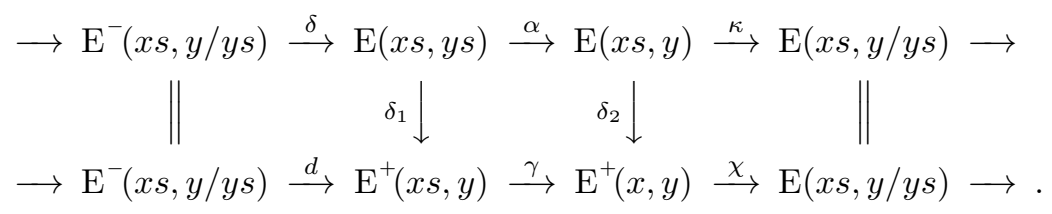

The following result is the twisted equivalent of [GJ, 5.2.3].

Proposition 3.2 Suppose that $x s>x$ and $y s<y$. For all $p$,

$$
e_{p}(x, y)-e_{p}(x s, y) \geq e_{p-1}(x s, y)-e_{p-1}(x s, y s)
$$

and this is an equality if, and only if, $\operatorname{Ker} d^{p-1}=\operatorname{Ker} \delta^{p-1}$ and $\operatorname{Ker} d^{p-2}=\operatorname{Ker} \delta^{p-2}$.

Proof. Since $d=\delta_{1} \delta, \operatorname{Ker} \delta \subseteq \operatorname{Ker} d$. Identify $\mathrm{E}$ with $\mathrm{E}^{p-1}$ and $d$ with $d^{p-2}$ in diagram (3.1.1). Because the second row is exact, there is a short exact sequence

$$
0 \longrightarrow \operatorname{Im} d^{p-2} \longrightarrow \mathrm{E}^{p}(x s, y) \longrightarrow \mathrm{E}^{p}(x, y) \longrightarrow \operatorname{Ker} d^{p-1} \longrightarrow 0
$$

Then

$$
\begin{aligned}
e_{p}(x, y)-e_{p}(x s, y) & =\operatorname{dim} \operatorname{Ker} d^{p-1}-\left(e_{p-2}(x s, y / y s)-\operatorname{dim} \operatorname{Ker} d^{p-2}\right) \\
& \geq \operatorname{dim} \operatorname{Ker} \delta^{p-1}-\left(e_{p-2}(x s, y / y s)-\operatorname{dim} \operatorname{Ker} \delta^{p-2}\right) \\
& =e_{p-1}(x s, y)-e_{p-1}(x s, y s),
\end{aligned}
$$

where the last equality uses the exactness of the first row of (3.1.1).

Corollary $3.3[\mathrm{C}, 3.9]$ Suppose that $x s>x, y s<y$, and $x s \nless y s$. For all $p$,

$$
e_{p}(x, y)=e_{p}(x s, y)+e_{p-1}(x s, y) .
$$

Proof. Because $\mathrm{E}(x s, y s)=0, \delta=0$ and $d=0$. The conditions for equality in 3.2 are satisfied.

These results led naturally to the conjecture that $e_{p}=r_{p}$ for all $p[\mathrm{C}, 3.1]$. It was soon discovered that there are examples where $r_{p}(x, y)$ is negative [Boe], so equality in 3.2 can not hold in general. One easy consequence of [GJ, 5.2.3] is that $r_{1}$ is, at least, a lower bound for $e_{1}$. (Later, it will be shown that $e_{1} \neq r_{1}$.)

PRoposition $3.4 e_{1} \geq r_{1}$

Proof. Assume there is a counterexample, $e_{1}(x, y)<r_{1}(x, y)$, with $x$ maximal in the Bruhat ordering. If $x=w_{0}, e_{1}\left(w_{0}, y\right)=0=r_{1}\left(w_{0}, y\right)$ so $x<w_{0}$. Choose an $s \in S$ with $x s>x$. There are two cases to consider.

If $y s>y$, then $e_{1}(x, y)=e_{1}(x s, y s)$ by 3.1. Since $x$ is maximal, $e_{1}(x s, y s) \geq$ $r_{1}(x s, y s)=r_{1}(x, y)$ by equation (3.0.2).

If $y s<y$, then 3.2 implies that $e_{1}(x, y) \geq e_{1}(x s, y)+e_{0}(x s, y)-e_{0}(x s, y s)$. Since $e_{0}=r_{0}$ and $x$ is maximal, $e_{1}(x, y) \geq r_{1}(x s, y)+r_{0}(x s, y)-r_{0}(x s, y s)=r_{1}(x, y)$ by (3.0.1).

In either case, $e_{1}(x, y) \geq r_{1}(x, y)$, which contradicts the choice of $x$.

The twisted sequence in diagram (3.1.1) has the same terms as the two-line spectral sequence of [C, 3.4]. It is an indirect resolution of the conjecture that the coboundary of the spectral sequence should factor as $d=\delta_{1} \delta$ [C, p. 37]. It can also 
be substituted for the spectral sequence in many of the proofs. As an example, one result that is needed below will be re-proved here.

Proposition 3.5 [C, 3.8] If $x \leq y$ and $n=\ell(x, y)$, then $e_{n}(x, y)=1$.

Proof. Suppose that $x \leq y$ and assume that there is a counterexample with $x$ maximal. If $x=w_{0}$, then $y=w_{0}, n=0$ and $e_{0}\left(w_{0}, w_{0}\right)=1$ so $x<w_{0}$. Choose an $s \in S$ so that $x s>x$. There are two cases to consider.

If $y s>y$, and $e_{n}(x, y)=e_{n}(x s, y s)$ by 3.1. Because $x$ is maximal and $x s \leq y s$, $e_{n}(x s, y s)=1$.

If $y s<y$, then consider diagram (3.1.1) with $\mathrm{E}=\mathrm{E}^{n-1}$ and apply the vanishing properties.

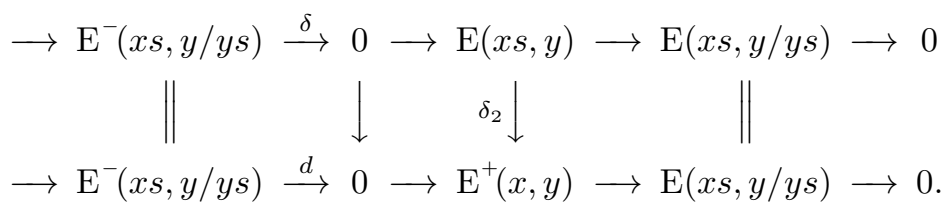

Then $\delta_{2}$ is an isomorphism, so $e_{n}(x, y)=e_{n-1}(x s, y)$. But $e_{n-1}(x s, y)=1$ since $x s \leq y$ and $x$ is maximal.

In either case, $e_{n}(x, y)=1$, which contradicts the choice of $x$.

In the remainder of this section, the recursive calculation of $e_{n-1}(x, y)$ where $n=$ $\ell(x, y)$ will be considered. Suppose that $x<x s<y s<y$ for some $s \in S$. Applying diagram (3.1.1) with $\mathrm{E}=\mathrm{E}^{n-2}$ yields

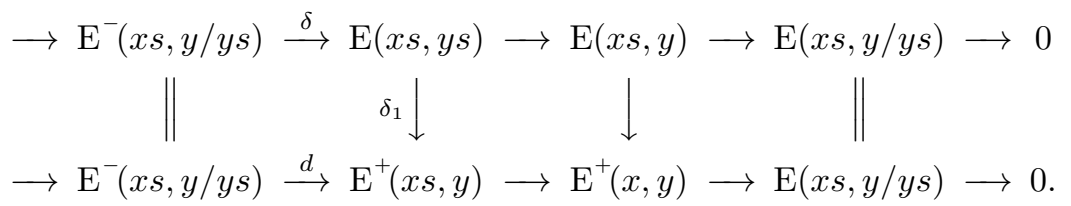

By $3.5, e_{n-2}(x s, y s)=e_{n-1}(x s, y)=1$ so that $\delta_{1}$ is an isomorphism or zero. But $\delta_{1}$ is part of the exact sequence

$$
\mathrm{E}^{n-2}(x s, y s) \stackrel{\delta_{1}}{\longrightarrow} \mathrm{E}^{n-1}(x s, y) \longrightarrow \mathrm{E}^{n-1}\left(M_{x s}, \theta M_{y}\right) \longrightarrow 0,
$$

showing that $\delta_{1}$ is an isomorphism, if and only if, $\mathrm{E}^{n-1}\left(M_{x s}, \theta M_{y}\right)$ is zero. By the adjoint pairing $(T, R), \mathrm{E}^{n-1}\left(M_{x s}, \theta M_{y}\right)$ is isomorphic to $\mathrm{E}^{n-1}\left(T M_{x s}, T M_{y}\right)$. The vanishing behavior of this singular extension group determines whether $d$ is zero or surjective. This suggests a conjecture on singular vanishing.

Conjecture 3.6 If $x<x s<y s<y$, then $\mathrm{E}^{n-1}\left(T M_{x s}, T M_{y}\right)=0$, where $n=\ell(x, y)$.

Proposition 3.7 Suppose that $x<y$ and let $n=\ell(x, y)$. Conjecture 3.6 implies that

$$
e_{n-1}(x, y)=r_{1}(x, y) .
$$

Proof. Assume there is a counterexample with $x$ maximal. Because $y \leq w_{0}, x<w_{0}$ and there is an $s \in S$ with $x s>x$. There are three cases to consider.

If $y s>y, 3.1$ implies that $e_{n-1}(x, y)=e_{n-1}(x s, y s)$. Since $x$ is maximal and $x s<y s, e_{n-1}(x s, y s)=r_{1}(x s, y s)=r_{1}(x, y)$ by equation (3.0.2). 
If $y s<y$ and $x s \nless y s, e_{n-1}(x, y)=e_{n-1}(x s, y)+e_{n-2}(x s, y)$ by 3.3. Since $x s \leq y$, $e_{n-1}(x s, y)=1$ by 3.5. If $x s=y$, then $n=1$ and $e_{0}(x, y)=r_{0}(x, y)=r_{1}(x, y)$ so $x s<y$ by the choice of $x$. Because $x$ is maximal, $e_{n-2}(x s, y)=r_{1}(x s, y)$. Then $e_{n-1}(x, y)=1+r_{1}(x s, y)=r_{1}(x, y)$ by equation (3.0.2).

If $x<x s<y s<y$ and assuming that conjecture 3.6 is true, $\delta_{1}$ in diagram (3.5.1) is an isomorphism. Then $e_{n-1}(x, y)=e_{n-2}(x s, y)$. Because $x$ is maximal, $e_{n-2}(x s, y)=r_{1}(x s, y)=r_{1}(x, y)$ by equation $(3.0 .2)$.

In each case, $e_{n-1}(x, y)=r_{1}(x, y)$, which contradicts the choice of $x$.

\section{Applichtions in CAtegory $\mathcal{O}$ : Younger Results}

Most of the results of the last section have been known for a long time. The newer results involve $r_{1}$. The first new result in this direction was published by Mazorchuk in 2007.

Proposition 4.1 [Maz, Lemma 33] $e_{1}\left(1, w_{0}\right)=|S|$.

Corollary 4.2 For all $x, y \in W$,

(i) $e_{1}\left(x, w_{0}\right)=r_{1}\left(x, w_{0}\right)$ and

(ii) $e_{1}(1, y)=r_{1}(1, y)$.

The first item of 4.2 is equivalent to the original statement of [Maz, Theorem 32] (adjusting for anti-dominance and ignoring the grading). It is expressed here in terms of $r_{1}$. The proof of the corollary uses the following lemma.

Lemma 4.3 Suppose that $x s>x$ and $y s<y$ for some $s \in S$. If $e_{1}(x, y)=r_{1}(x, y)$, then $e_{1}(x s, y)=r_{1}(x s, y)$

Proof. Suppose that $e_{1}(x s, y) \neq r_{1}(x s, y)$. By $3.4, e_{1}(x s, y)>r_{1}(x s, y)$. Using 3.2 and 3.0.1,

$$
\begin{aligned}
e_{1}(x, y) & \geq e_{1}(x s, y)+e_{0}(x s, y)-e_{0}(x s, y s) \\
& >r_{1}(x s, y)+r_{0}(x s, y)-r_{0}(x s, y s)=r_{1}(x, y),
\end{aligned}
$$

so $e_{1}(x, y) \neq r_{1}(x, y)$

Proof of the corollary. To show that $e_{1}\left(1, w_{0}\right)=r_{1}\left(1, w_{0}\right)$, apply [Hum1, 7.10(20)] with $x=1$ and $w=w_{0}$ to get

$$
\sum_{1 \leq y \leq w_{0}} R_{1, y}=q^{n}
$$

where $n=\ell\left(1, w_{0}\right)$. The coefficient of $q^{n-1}$ on the left-hand side is

$$
(-1)^{1} r_{n-1}\left(1, w_{0}\right)+|S|,
$$

so $r_{1}\left(1, w_{0}\right)=r_{n-1}\left(1, w_{0}\right)=|S|$.

To prove item (i), assume that there is a counterexample with $x$ minimal. Then $x>1$ and there is an $s \in S$ with $x s<x$. By minimality of $x, e_{1}\left(x s, w_{0}\right)=r_{1}\left(x s, w_{0}\right)$. The lemma implies that $e_{1}\left(x, w_{0}\right)=r_{1}\left(x, w_{0}\right)$, contradicting the choice of $x$.

The proof of item (ii) is similar. 
The next development was Noriyuki Abe's preprint that originally appeared on the ArXiv in 2010. Let $v(x, y)=e_{1}(x, y)-e_{0}\left(x, w_{0} / y\right)$ if $x \leq y$ and let $v(x, y)=0$ if $x \not \leq y$. If $x \leq y$, then $v(x, y)=\operatorname{dim} V\left(w_{0} x, w_{0} y\right)$ in Abe's notation. Then [Abe1, theorem 4.4] becomes $v=r_{1}$. As stated, the theorem is not true. There are 16 pairs $(x, y)$ in type $\mathrm{B}_{3}$ with $r_{1}(x, y)=4$ but, by definition, $v \leq 3$ [Abe1, Theorem 1.1(1)]. Abe's recursion for $V$ [Abe1, Theorem 4.3] does imply that $v \leq r_{1}$ (by comparison with 3.0.2). Then, combined with $3.4, v \leq r_{1} \leq e_{1}$ or

$$
r_{1}(x, y) \leq e_{1}(x, y) \leq r_{1}(x, y)+e_{0}\left(x, w_{0} / y\right)
$$

Note that $e_{0}\left(1, w_{0} / y\right)=0$ and $e_{0}\left(x, w_{0} / w_{0}\right)=0$, so Abe's inequality does generalize 4.2. Although $v \neq r_{1}$, Abe has communicated an example in type $\mathrm{B}_{3}$ showing that $e_{1} \neq r_{1}[\mathrm{Abe} 2]$.

In the remainder, the twisted sequence approach will be used to prove properties of $v$ that correspond with Abe's results from [Abe1].

Proposition 4.4 If $x s>x$ and $y s<y$, then $e_{0}\left(x s, w_{0} / y\right)=e_{0}\left(x, w_{0} / y s\right)$.

Proof. Let $M=M_{x s}$ and $N=M_{w_{0}} / M_{y s}$. There is a commutative diagram with exact rows,

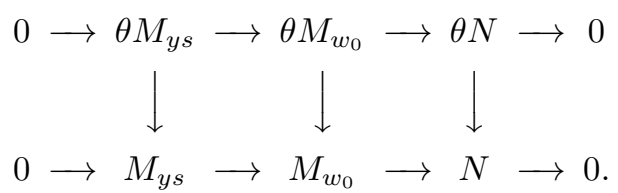

By the snake lemma, $K N=M_{w_{0}} / M_{y}$. By the adjoint pairing $(C, K), \mathrm{H}\left(M_{x s}, K N\right)$ and $\mathrm{H}\left(M_{x}, N\right)$ are isomorphic.

By 3.1, if $x s>x$ and $y s<y, e_{1}(x s, y)=e_{1}(x, y s)$ which proves the following property of $v$, which corresponds to [Abe1, 4.3(1)].

Corollary 4.5 If $x s>x$ and $y s<y$, then $v(x s, y)=v(x, y s)$.

Next, there is another ladder diagram that links extensions of fractional Verma modules to the twisted sequence.

Proposition 4.6 Suppose that $x s>x$ and $y s<y$. There is a commutative diagram with exact rows,

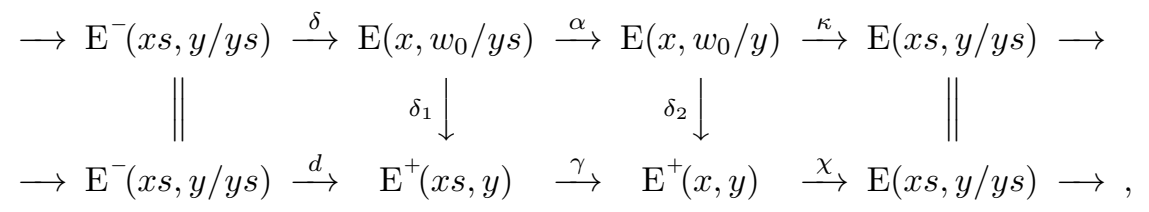

where the second row is the same as the second row of diagram (3.1.1).

Proof. The proof is similar in structure to the proof of 2.1. Fix a commuting triangle of Verma module injections,

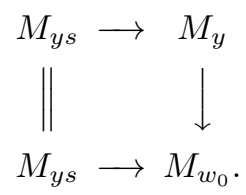


Diagram 1:

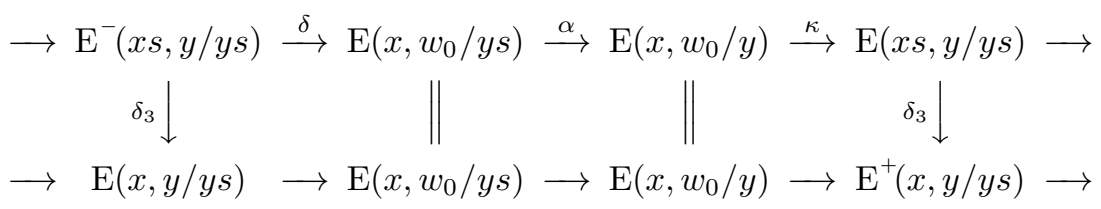

The map $\delta_{3}$ is the same as the isomorphism $\delta_{3}$ from diagram (2.1.2) with $M=M_{x s}$ and $N=M_{y}$. The second row is the long exact sequence associated to the exact sequence,

$$
M_{y} / M_{y s} \hookrightarrow M_{w_{0}} / M_{y s} \rightarrow M_{w_{0}} / M_{y} .
$$

Define $\delta$ and $\kappa$ so that the diagram commutes. This produces a commutative diagram with exact rows.

Diagram 2:

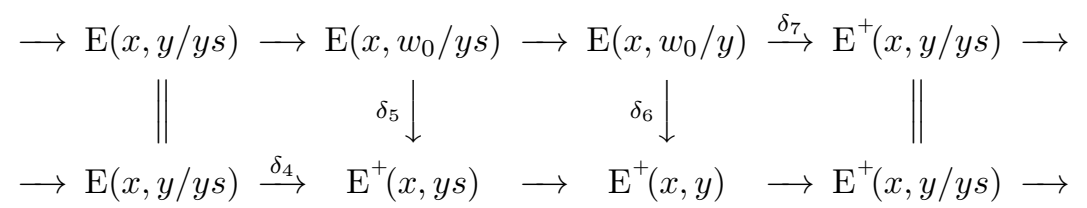

This is a commutative diagram with exact rows where $\delta_{k}, 4 \leq k \leq 7$ are natural connecting maps (all derived from rotations of diagram (4.6.1)). For example, the middle square commutes because of the short ladder,

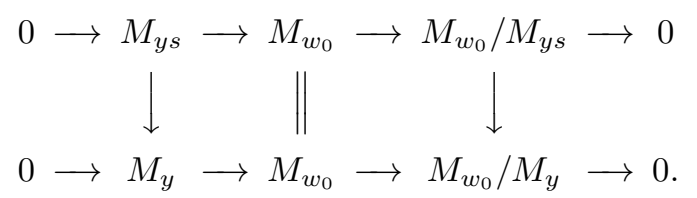

Diagram 3:

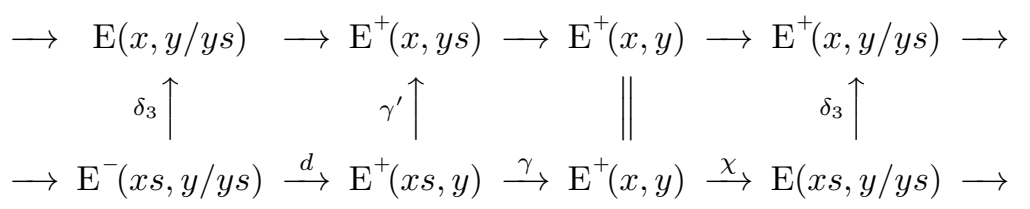

This is a commutative diagram with exact rows because it is diagram (2.1.3) with $M=M_{x s}$ and $N=M_{y}$. Since $\gamma^{\prime}$ is an isomorphism, assembling the diagrams completes the proof.

Applying the same argument as in the proof of 3.2 yields the following inequality.

Proposition 4.7 Suppose that $x s>x$ and $y s<y$. For all $p$,

$$
e_{p}(x, y)-e_{p}(x s, y) \geq e_{p-1}\left(x, w_{0} / y\right)-e_{p-1}\left(x, w_{0} / y s\right) .
$$

This is an equality if, and only if, $\operatorname{Ker} d^{p-1}=\operatorname{Ker} \delta^{p-1}$ and $\operatorname{Ker} d^{p-2}=\operatorname{Ker} \delta^{p-2}$. 
COROLlary 4.8 If $x s>x$ and $y s<y$, then

$$
e_{1}(x, y)-e_{1}(x s, y) \geq e_{0}\left(x, w_{0} / y\right)-e_{0}\left(x s, w_{0} / y\right)
$$

and this is an equality if, and only if, $\operatorname{Ker} d^{0}=\operatorname{Ker} \delta^{0}$

Proof. Taking $p=1$ in 4.7 ,

$$
e_{1}(x, y)-e_{1}(x s, y) \geq e_{0}\left(x, w_{0} / y\right)-e_{0}\left(x, w_{0} / y s\right) .
$$

By $4.4, e_{0}\left(x, w_{0} / y s\right)=e_{0}\left(x s, w_{0} / y\right)$.

The conclusion is equivalent to $v(x, y) \geq v(x s, y)$. When $x s<y s$, Abe proves $v(x, y)=v(x s, y)$ by showing that the images of $\mathrm{E}^{1}(x s, y)$ and $\mathrm{E}^{1}(x, y)$ in $\mathrm{E}^{1}\left(x, w_{0}\right)$ are the same [Abe1, 4.3(2)].

The preceding proposition is sufficient, by itself, to explain Abe's counter-example for $e_{1}=r_{1}$. In type $\mathrm{B}_{3}$, let $s_{1}, s_{2}$, and $s_{3}$ be the simple root reflections, where $s_{1} s_{2}$ has order 3 and $s_{2} s_{3}$ has order 4 . Take $x=s_{1} s_{3}, y=w_{0} s_{3}=s_{2} s_{3} s_{1} s_{2} s_{3} s_{2} s_{1} s_{2}$, and $s=s_{2}$. Using the work of $\mathrm{H}$. Matumoto [Mat] on scalar, generalized Verma module homomorphisms, Abe shows that there is a nonzero homomorphism between $M_{x}$ and $M_{w_{0}} / M_{y}$ so $e_{0}\left(x, w_{0} / y\right) \neq 0$ [Abe2]. Kazhdan-Lusztig multiplicities imply that $e_{0}\left(x, w_{0} / y\right)-e_{0}\left(x s, w_{0} / y\right)=1$. By $4.8, e_{1}(x, y)>e_{1}(x s, y)$, which means $e_{1}(x, y) \neq r_{1}(x, y)$.

Proposition 4.9 Suppose that $x<x s \leq y$ and $y s<y$. If $x s \nless y s$, then $v(x, y) \leq$ $v(x s, y)+1$ and this is an equality if, and only if, $\operatorname{Ker} \delta^{0}=0$.

Proof. In $4.6, d=0$ by 3.3. Also $e_{0}(x s, y / y s)=1$ implies that $e_{0}\left(x, w_{0} / y\right)-$ $e_{0}\left(x, w_{0} / y s\right) \leq 1$.

The condition for equality in 4.9 must somehow be equivalent to the condition $v_{s} \notin s V\left(w_{0} x s, w_{0} y\right)$ from [Abe1, 4.3(2)]. Finally, another twisted sequence can be used to prove a result that is also consistent with [Abe1, 4.3(2)].

Suppose that $x s>x$ and $y s<y$. Let $M=M_{x s}$ and $N=M_{w_{0}} / M_{y s}$. There is a twisted sequence associated to $N$. From diagram (4.4.1), $I N=M_{w_{0} s} / M_{y s}$ so, by 2.1 , there is a commutative diagram with exact rows,

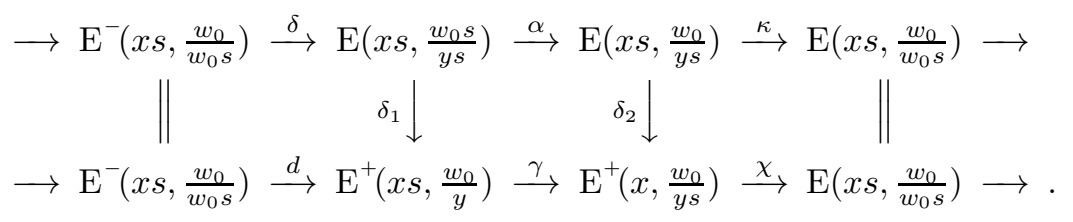

Proposition 4.10 Suppose that $x<x s \leq y$ and $y s<y$. If $x s \nless w_{0} s$, then $v(x, y)=$ $v(x s, y)+1$.

Proof. Because $y s<w_{0} s, x s \nless w_{0} s$ implies $x s \nless y s$ and hence $e_{0}\left(x s, w_{0} s / y s\right)=0$. If $\mathrm{E}$ is identified with $\mathrm{E}^{0}$ in diagram (4.9.1), $\kappa$ is an injective map, which implies that $\delta_{2}$ is injective. Working through the definitions, there is a commutative diagram,

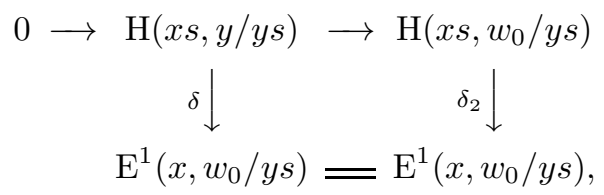


where $\delta$ is the homomorphism defined in the proof of 4.6. Since $\delta_{2}$ is injective, Ker $\delta=0$ and $v(x, y)=v(x s, y)+1$ by 4.9 .

In a similar vein, one can prove that $v(x, y)=v(x s, y)$ if $x<x s<y s<y$ and $e_{0}\left(x s, w_{0} s / y s\right)=0$. In that case, $e_{1}(x, y)=e_{1}(x s, y)$ as well.

If the goal is a general recursive formula for $e_{1}$, then the goal is well over the horizon. The classic conjecture, $e_{1}=r_{1}$, is false. Abe's recursion for $v$ is very effective (and $v$ is bounded above by the rank of $\mathfrak{g}$ ), but the resulting determination of $e_{1}$ depends on the very difficult problem of generalized Verma module homomorphisms. If $x \leq y$ and $e_{0}\left(x, w_{0} / y\right)$ is known, then $e_{1}(x, y)=v(x, y)+e_{0}\left(x, w_{0} / y\right)$.

\section{REFERENCES}

[Abe1] N. Abe, First extension groups of Verma modules and R-polynomials, J. Lie Theory 25 (2015), 377-393.

[Abe2] N. Abe, private communication, September 14, 2015.

[BGG] I. N. Bernstein, I. M. Gelfand and S. I. Gelfand, A category of $\mathfrak{g}$-modules, Func. Anal. Appl. 10 (1976), 87-92.

[Boe] B. D. Boe, A counterexample to the Gabber-Joseph conjecture, Kazhdan-Lusztig theory and related topics, Contemp. Math., , Amer. Math. Soc., Providence, R. I., 139 (1992), $1-3$.

[C] K. J. Carlin, Extensions of Verma modules, Trans. Amer. Math. Soc. 294, no. 1 (1986), 29-43.

[GJ] O. Gabber and A. Joseph, Towards the Kazhdan-Lusztig conjecture, Ann. Sci. Ecole Norm. Sup. 14 (1981), 261-302.

[Hum1] J. E. Humphreys, Reflection Groups and Coxeter Groups, Cambridge Studies in Advanced Math. 29, Cambridge University Press, Cambridge, 1990.

[Hum2] J. E. Humphreys, Representations of Semisimple Lie Algebras in the BGG Category $\mathcal{O}$, Grad. Studies in Math. 94, Amer. Math. Soc., Providence, R. I., 2008.

[Mat] H. Matumoto, The homomorphisms between scalar generalized Verma modules, Compos. Math. 150, no. 5 (2014), 877-892.

[Maz] V. Mazorchuk, Some homological properties of the category $\mathcal{O}$, Pacific Jour. of Math. 232 (2007), 313-341.

[W] C. A. Wiebel. An Introduction to Homological Algebra, Cambridge Studies in Advanced Mathematics 38, Cambridge University Press, 1994.

Department of Mathematics and Computer Science, Assumption College, 500 SalisBURY St., Worcester MA 01609-1296

E-mail address: kcarlin@assumption.edu 